\title{
Highly Anisotropic Thermal Transport in $\mathrm{LiCoO}_{2}$
}

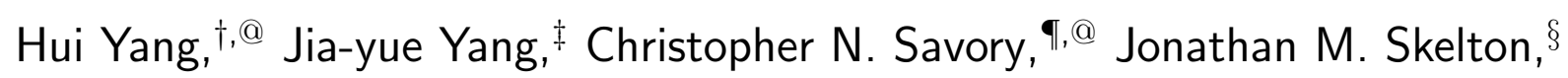
Benjamin J. Morgan,",@ David O. Scanlon,, , , $@$ and Aron Walsh*,†,\#,@

$\dagger$ Department of Materials, Imperial College London, Exhibition Road, London SW7 2AZ, $U K$

$\ddagger$ School of Energy and Power Engineering, Shandong University, Qingdao 266237, China I University College London, Department of Chemistry and Thomas Young Centre, 20 Gordon Street, London WC1H OAJ, UK $\S$ School of Chemistry, University of Manchester, Oxford Road, Manchester M13 9PL, UK

\|Department of Chemistry, University of Bath, Claverton Down, Bath BA2 7AY, UK $\perp$ Diamond Light Source Ltd., Diamond House, Harwell Science and Innovation Campus, Didcot, Oxfordshire OX11 ODE, UK

\#Department of Materials Science and Engineering, Yonsei University, Seoul 03722, Korea @ The Faraday Institution, Quad One, Harwell Science and Innovation Campus, Didcot, $U K$

E-mail: a.walsh@imperial.ac.uk

\begin{abstract}
$\mathrm{LiCoO}_{2}$ is the prototypical cathode in lithium-ion batteries. Its crystal structure consists of $\mathrm{Li}^{+}$and $\mathrm{CoO}_{2}{ }^{-}$layers that alternate along the hexagonal $\langle 0001\rangle$ axis. It is well established that the ionic and electronic conduction are anisotropic, but little is known regarding the heat transport. We analyse the phonon dispersion and lifetimes using anharmonic lattice dynamics based on quantum-chemical force constants. Around room temperature, the thermal conductivity in the hexagonal $a b$ plane of the
\end{abstract}


layered cathode is $\approx 6$ times higher than that along the $c$ axis. An upper limit to the average thermal conductivity at $\mathrm{T}=300 \mathrm{~K}$ of $38.5 \mathrm{~W} \mathrm{~m}^{-1} \mathrm{~K}^{-1}$ is set by short phonon lifetimes associated with anharmonic interactions within the octahedral face-sharing $\mathrm{CoO}_{2}{ }^{-}$network. Observations of conductivity $<10 \mathrm{~W} \mathrm{~m}^{-1} \mathrm{~K}^{-1}$ can be understood by additional scattering channels including grain boundaries in polycrystalline samples. The impact on thermal processes in lithium ion batteries is discussed.

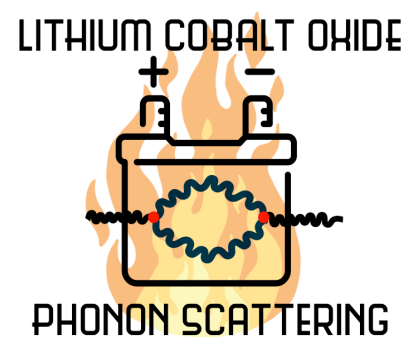


Lithium cobalt oxide, $\mathrm{LiCoO}_{2}$, has been widely used as a cathode material in lithium ion batteries (LIBs) following its discovery in $1980^{1}$ and its commercialization in the 1990s. The compound offers a number of attractive features including ease of synthesis, reversible lithium insertion, and high specific energy density. ${ }^{2,3} \mathrm{LiCoO}_{2}$ crystallizes in a layered structure (spacegroup R⿳亠丷⿵冂丶 , Figure 1a) in which $\mathrm{CoO}_{6}$ and $\mathrm{LiO}_{6}$ octahedra form parallel slabs. This layered structure means the material is effectively a 2 D ionic and electronic conductor. ${ }^{4,5}$

Co can be partially replaced by the transition metals $\mathrm{Ni}$ and Mn to form the NMC system $\mathrm{LiNi}_{x} \mathrm{Mn}_{y} \mathrm{Co}_{z} \mathrm{O}_{2}(x+y+z=1)$. The Co:Ni:Mn ratio can be tuned to optimize the mechanical and electrochemical performance while reducing cost. ${ }^{6,7}$ Many NMC compositions preserve the same structure type, with some local Jahn-Teller distortions depending on the combination of oxidation states present in the mixed-metal system. ${ }^{6,8}$

One key challenge for LIBs is to improve lifetimes and safety characteristics without compromising energy and power densities. ${ }^{9,10}$ Thermal management is an important issue and is intimately connected to chemical degradation and battery lifetime. There is a significant gap in knowledge of battery manufacturers and developers on how temperature and thermal management affects capacity/power fade, thermal runaway, and other key characteristics of Li-ion cells. ${ }^{11}$ With modern computational techniques many key battery processes can be accurately described by computer simulations, including the defect chemistry and associated ionic and electronic transport. ${ }^{12}$ However, little is known regarding heat transport. Very recent work has explored thermal conductivity of battery anode ${ }^{13,14}$ and cathode ${ }^{15}$ materials using molecular dynamics simulations.

In this Letter, we assess the lattice dynamics and thermal transport of $\mathrm{LiCoO}_{2}$ within phonon many-body perturbation theory. For the harmonic phonon dispersion, we find a sensitivity to the choice of the density-functional theory (DFT) electron exchange and correlation functional. Using the HSE06 hybrid functional with the Tkatchenko-Scheffler dispersion correction, we calculate the lifetime and heat conduction of each phonon mode to evaluate the modal contributions to the macroscopic lattice thermal conductivity. We predict a value 
(a)

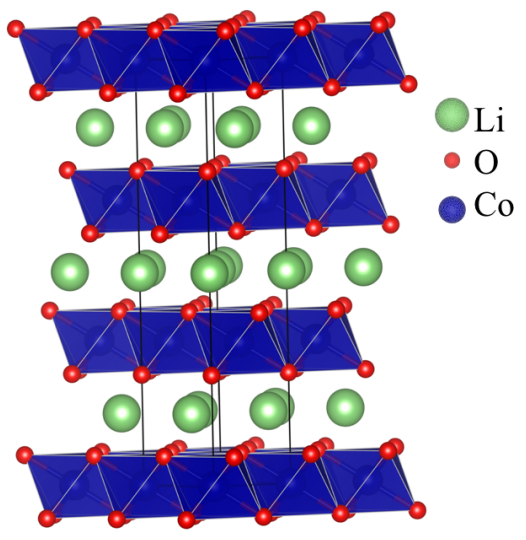

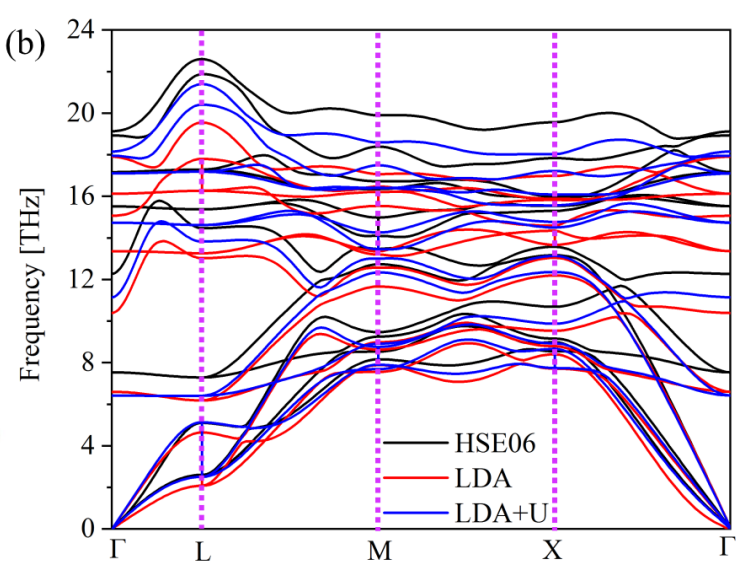

Figure 1: (a) Layered crystal structure of $\mathrm{LiCoO}_{2}$ with spacegroup $\mathrm{R} \overline{3} \mathrm{~m}$, where the stacking is along the hexagonal $c$ axis. This image was generated using VESTA. ${ }^{16}$ (b) Calculated phonon dispersion of $\mathrm{LiCoO}_{2}$ using three DFT exchange-correlation functionals and a $3 \times 3 \times 3$ supercell to calculate the harmonic force constants.

of $53.6 \mathrm{~W} \mathrm{~m}^{-1} \mathrm{~K}^{-1}$ (hexagonal $a b$ plane) and $8.4 \mathrm{~W} \mathrm{~m}^{-1} \mathrm{~K}^{-1}$ (c axis) at room temperature in the pristine $\mathrm{LiCoO}_{2}$ crystal. The origin and impact of this large anisotropy is explored.

Phonon dispersion. The harmonic phonon dispersion of $\mathrm{LiCoO}_{2}$ is shown in Figure $1 \mathrm{~b}$. The positive (real) frequencies across the vibrational Brillouin zone confirm a dynamically stable structure. The four atoms in the primitive (rhombohedral setting) unit cell result in $12(3 N)$ phonon modes. A group theoretic analysis within the $\mathrm{D}_{3 d}$ point group assigns the irreducible representations of the acoustic $\left(A_{2 u}+E_{u}\right)$ and optic branches $\left(A_{1 g}+2 A_{2 u}+2 E_{u}+\right.$ $E_{g}$ ). Of these, the $2 A_{2 u}+2 E_{u}$ modes are infra-red (IR) active and the $A_{1 g}+E_{g}$ modes are Raman active.

The low-spin $\mathrm{d}^{6}$ configuration of $\mathrm{Co}(\mathrm{III})$ in pristine $\mathrm{LiCoO}_{2}$ avoids the more complex electronic structures associated with the $\mathrm{Co}(\mathrm{II})$ and $\mathrm{Co}(\mathrm{IV})$ species. However, we still expect some sensitivity to the choice of DFT exchange-correlation functional, as the localized wavefunctions of $3 \mathrm{~d}$ transition metals are susceptible to self-interaction errors. ${ }^{17}$

The LDA and LDA $+U$ functionals produce qualitatively similar phonon dispersion, in agreement with the work of Du and co-workers. ${ }^{8}$ However, the inclusion of non-local exchange with HSE06 and the Tkhatchenko-Scheffler dispersion to describe the interlayer interactions results in a stiffening of the frequencies of the optic branches (also observed in other dielectric 
materials $\left.{ }^{18}\right)$. A comparison with available experimental data is given in Table 1. For example, IR measurements place an $E_{u}$ mode at $7.8 \mathrm{THz}$, which compares to $7.6 \mathrm{THz}$ from HSE06, while $\mathrm{LDA} / \mathrm{LDA}+U$ predict a value at $\sim 6 \mathrm{THz}$. Care should however be taken with direct comparison with experiment as the predictions do not include finite-temperature effects such as thermal expansion and higher-order anharmonic frequency shifts due to phonon-phonon interactions. We would generally expect volume expansion at finite temperature to soften the phonon frequencies, and hence our calculated frequencies to be overestimated compared to the room-temperature measurements.

Table 1: Comparison of phonon frequencies for selected modes at the $\Gamma$ point in the vibrational Brillouin zone of $\mathrm{LiCoO}_{2}$. The five rows compare the calculated frequencies $(\mathrm{THz})$ obtained with four different DFT exchange-correlation functionals (TS denotes the TkatchenkoScheffler dispersion correction) to room-temperature IR and Raman spectra.

\begin{tabular}{ccccc}
\hline Method & $A_{1 g}$ & $E_{g}$ & $A_{2 u}$ & $E_{u}$ \\
\hline LDA & 18.09 & 14.30 & 12.25 & 7.64 \\
LDA+U & 17.95 & 14.73 & 11.14 & 6.42 \\
HSE06 & 19.12 & 15.52 & 12.27 & 7.53 \\
HSE06+TS & 19.17 & 15.62 & 12.81 & 7.56 \\
Exp. & $17.87^{a}$ & $14.57^{a}$ & $12.59^{b}$ & $7.80^{b}$ \\
\hline${ }^{a}$ Raman active modes ${ }^{19}{ }^{b} \mathrm{IR}$ active modes
\end{tabular}

Lattice thermal conductivity. The lattice thermal conductivity of a crystalline material $(\kappa)$ depends on the nature of its heat-carrying vibrations. Formally, each phonon mode makes a contribution to the macroscopic thermal conductivity given by the product of the modal heat capacity $\left(C_{V}\right)$, the group velocity $(v)$, and the phonon mean free path $(v \times \tau$, where $\tau$ is the phonon lifetime). The macroscopic $\kappa$ is obtained by summing over band indices $(v)$, averaging over wavevectors $(q)$ and normalizing by the cell volume:

$$
\kappa=\frac{1}{N V_{0}} \sum_{q v} C_{V, q v} v_{q v} \otimes v_{q v} \tau_{q v}
$$

where $N$ is the number of unit cells in the crystal (equivalent to the number of wavevectors included in the Brillouin zone summation) and $V_{0}$ is the volume of the crystallographic unit cell. The heat capacity and group velocity can be determined from the harmonic phonon 
dispersion (Figure 1b), but lifetime is ill-defined (infinite) in the harmonic approximation due to absence of phonon-phonon interactions.

We calculate the lifetime of each phonon mode within phonon many-body perturbation theory by taking into account the leading term of three-phonon scattering processes. ${ }^{21}$ Our calculated thermal conductivity is plotted in Figure 2. The conductivity decays exponentially with increasing temperature and is found to be highly anisotropic: around room temperature, the thermal conductivity in the hexagonal $a b$ plane is $53.6 \mathrm{~W} \mathrm{~m}^{-1} \mathrm{~K}^{-1}$, while the value along the $c$ axis is $8.4 \mathrm{~W} \mathrm{~m}^{-1} \mathrm{~K}^{-1}$.

The experimentally measured thermal conductivity is as low as $4 \mathrm{~W} \mathrm{~m}^{-1} \mathrm{~K}^{-1}$ at $280 \mathrm{~K},{ }^{22}$ which is slightly below our calculated result along the $c$ axis. Our predictions should represent an upper limit for pristine single crystals, as in real, imperfect samples other scattering processes occur due to extended (e.g. dislocations and grain boundaries) and point (e.g. $\mathrm{Li}_{\mathrm{Co}}$ antisite) defects. Indeed, recent classical molecular dynamics simulations using empirical potentials predict that polycrystalline $\mathrm{LiCoO}_{2}$ has a greatly reduced thermal conductivity. ${ }^{15}$ We find that the experimental value of thermal conductivity in polycrystalline samples can be reproduced if the phonon mean-free path length is limited to a threshold value of $2 \mathrm{~nm}$ (see Figure 2).

Origin of anisotropic transport. Phonon lifetimes and mean free paths have only recently become accessible to first-principles calculations, and the majority of available data is on face-centred cubic systems. ${ }^{21,23,24}$ In comparison to tetrahedral semiconductors with simpler structures such as GaAs and CdTe, ${ }^{25}$ the average strength of the three-phonon interactions in $\mathrm{LiCoO}_{2}$ is three orders of magnitude larger, and the associated phonon lifetimes of $\mathrm{LiCoO}_{2}$ are correspondingly three orders of magnitude shorter (see Figure 3). To investigate the origin of the directional anisotropy, we analyzed separately the model contributions to the thermal conductivity. The analysis in Figure3 shows that the modes that make the largest contributions to the thermal transport are those that have faster group velocities (panel a and b) and longer modal lifetimes (panel c and d), and hence longer mean-free paths (panel e 


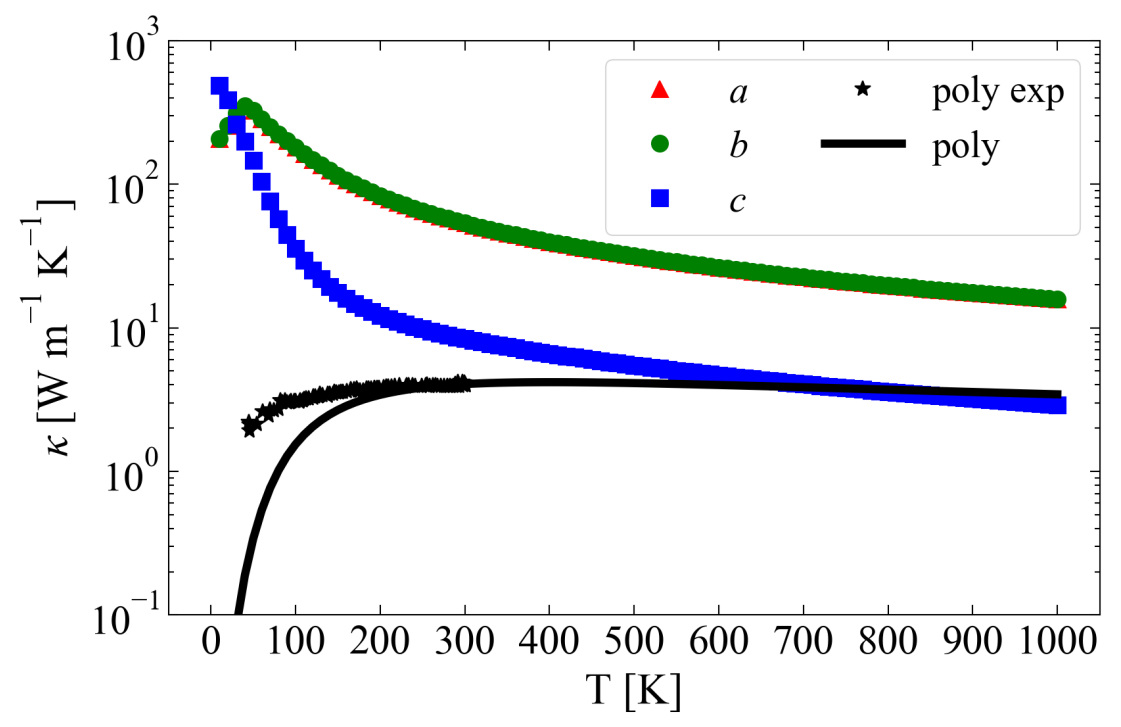

Figure 2: Calculated lattice thermal conductivity $(\kappa)$ of $\mathrm{LiCoO}_{2}$ along the $a, b$ and $c$ axes. The corresponding modal accumulation functions are presented in Supporting Information. The experimental data ${ }^{22}$ was measured on polycrystalline samples using a steady-state technique (poly exp). The black line is a simulation of a polycrystalline (poly) film of $\mathrm{LiCoO}_{2}$ with $\kappa_{\text {average }}=\frac{1}{3}\left(\kappa_{a}+\kappa_{b}+\kappa_{c}\right)$ and a boundary-scattering model that limits the mean free path to an average grain size; the experimental measurements are well reproduced with a limit of $2 \mathrm{~nm}$.

and $\mathrm{f}$ ). The longest calculated mean-free path of the heat carrying modes is $44 \mathrm{~nm}$ in the $a b$ plane and $446 \mathrm{~nm}$ along the $c$ axis in the perfect crystal. However, the thermal conductivity is a summation over the phonon Brillouin zone (c.f. Equation 1), and the lower density of long-lived modes along the $c$ axis results results in smaller average path lengths of $1.6 \mathrm{~nm}$ compared to $3.9 \mathrm{~nm}$ in the $a b$ plane.

Heat generation during the charge-discharge process of a battery can exceed $1 \mathrm{~W} / \mathrm{cm}^{3}$ depending on the cycling rate. This is due to a combination of resistive heating from ion and electron transport together with changes in entropy. ${ }^{26}$ Simulations of battery components typically assume isotropic thermal transport, which we have shown is not the case. The thermal stability of $\mathrm{LiCoO}_{2}$ is known to be poor, which can lead to thermal runaway under high-temperature operation or overcharging. ${ }^{27}$ This could be avoided by tuning the grain size distributions in combination with active binders that act as heat sinks in a composite 

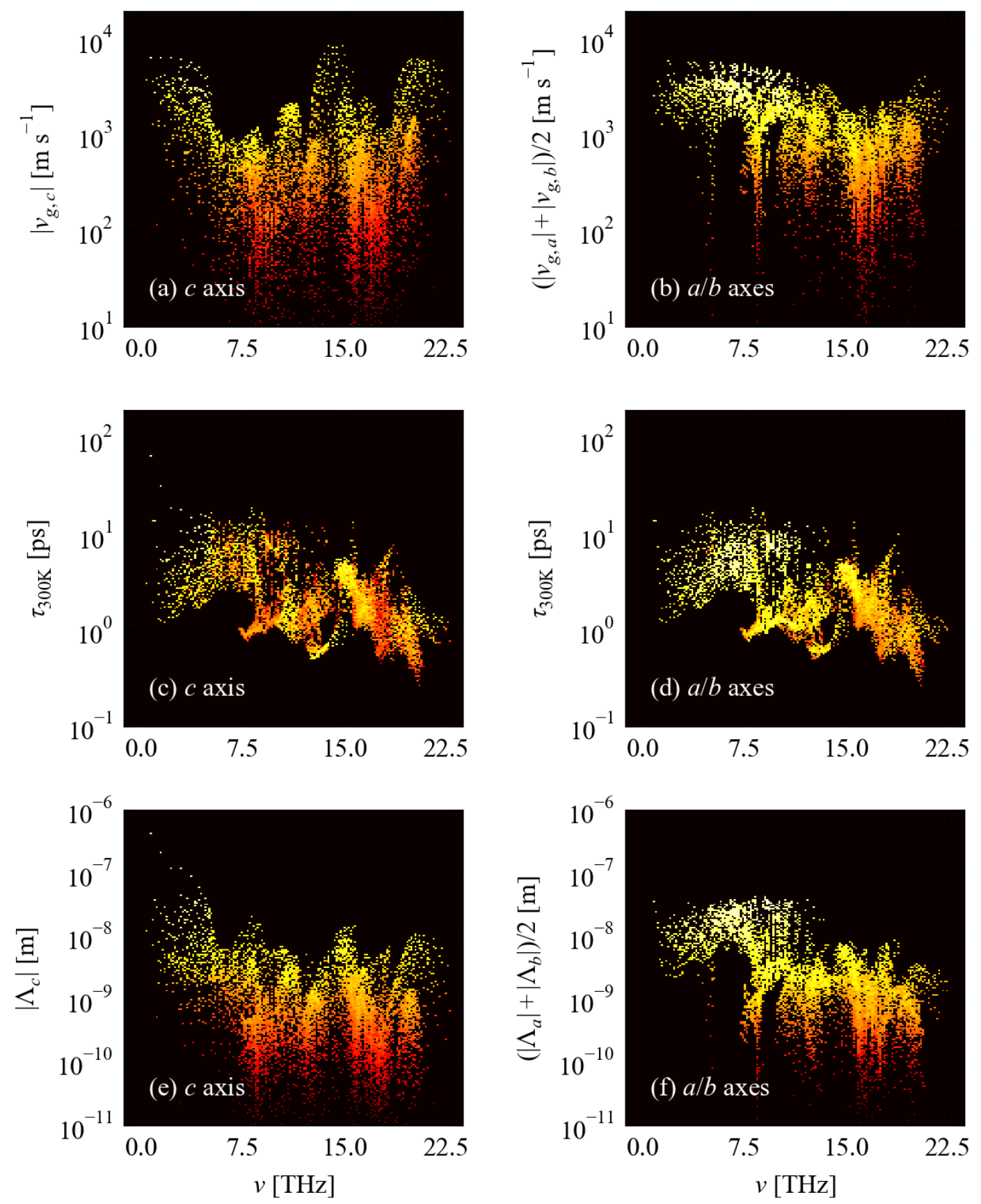

Figure 3: Analysis of the thermal conductivity of $\mathrm{LiCoO}_{2}$. The left- and right-hand columns show transport along the $c$ direction and in the $a b$ plane respectively. Subplots (a) and (b) show the spectrum of group velocities, subplots (c) and (d) show the modal lifetimes, and subplots (e) and (f) show the mean-free paths. The heat maps are colour coded by the modal contributions to $\kappa$, from red (low) to yellow (high). 
electrode structure.

The lattice thermal conductivity of $\mathrm{NMC}$ compositions will be even lower than $\mathrm{LiCoO}_{2}$ owing to the additional phonon-scattering from cation disorder and the associated spatial inhomogeneity in the phonon frequencies and lifetimes. This also introduces a technical challenge for modelling, which would need to consider an ensemble of transition metal and oxidation state distributions for force constant calculations. Beyond shorter phonon lifetimes, however, cation disorder may also provide a kinetic barrier to the formation of secondary phases, which could account for the overall improved stability of the NMC compositions.

The impact of thermal effects on anion redox has recently been highlighted, where heat dissipation contributes to voltage hysteresis upon battery cycling. ${ }^{28}$ Investigation of the coupling between the vibrational modes of defects with bulk phonon transport is a worthwhile direction for future research.

To conclude, the thermal conductivity in $\mathrm{LiCoO}_{2}$ is highly anisotropic, with a $6 \times$ larger $\kappa$ in the in-plane direction. First-principles analysis of the harmonic phonon dispersion and anharmonic phonon-phonon interactions reveal that the anisotropy largely originates from differences in the group velocity of the heat-carrying phonon modes, in particular the number of modes with larger in-plane than out-of-plane velocities. We found that the hybrid HSE06 exchange-correlation functional provides a good description of the vibrational properties of $\mathrm{LiCoO}_{2}$ and will likely be essential for the commercial NMC system where the mixedoxidation states of $\mathrm{Ni}, \mathrm{Mn}$ and Co exhibit strong electron correlation. Our study provides a robust foundation for advancing our understanding of thermal processes in battery materials.

Computational methods. The starting point for the structural relaxation was the experimentallydetermined structure of $\mathrm{LiCoO}_{2}$ from the work of Cava and co-workers. ${ }^{29}$ The total energy and electronic structure calculations were performed using plane-wave density-functional theory as implemented in the Vienna Ab initio Simulation Package. ${ }^{30}$ A plane-wave basis set with a $600 \mathrm{eV}$ cutoff was used to expand the electronic wavefunctions. Structural relaxation was performed with a $6 \times 6 \times 6 k$-point mesh, for which the total energy was converged to 
within $1 \mathrm{meV}$ per atom, and a force tolerance of $0.001 \mathrm{eV} / \AA$ was set for convergence of the ion positions to ensure accurate atomic positions and lattice parameters.

To account for the weak inter-layer interactions in $\mathrm{LiCoO}_{2}$ we also tested the TkatchenkoScheffler dispersion ${ }^{31}$ with Hirshfeld partitioning, ${ }^{32}$ which has been shown to reproduce the experimental lattice parameters ${ }^{29}$ as well as the Li-intercalation energies. ${ }^{33,34}$ For LDA $+U$, a representative $U$ value of $3.9 \mathrm{~V}$ was applied to the Co d orbitals. The hybrid HSE06 ${ }^{35}$ functional was used in both harmonic and anharmonic lattice-dynamics calculations.

The second-order (harmonic) force constants were computed using a $3 \times 3 \times 3$ supercell expansion and a finite-difference displacement step of $10^{-2} \AA$. The third-order (anharmonic) force constants were also computed using $3 \times 3 \times 3$ expansion but using a larger $3 \times 10^{-2} \AA$ step. The lattice thermal conductivity of $\mathrm{LiCoO}_{2}$ was obtained by solving the phonon Boltzmann transport equation within the relaxation-time approximation using the Phono3py package. ${ }^{36}$

Data access statement: The associated structure files and input/output of the phonon calculations are available in an online data repository: [link to be updated upon acceptance].

\section{Acknowledgement}

This work was carried out with funding from the Faraday Institution (faraday.ac.uk; EP/S003053/1) grant number FIRG003, and used the MICHAEL computing cluster. We are also grateful for computer resources from RWTH Aachen. JY is supported by Shandong University (Qilu Young Scholar 89963031). AW and BJM are supported by Royal Society University Research Fellowships (Nos. UF100278 and UF130329). JMS is supported by a Presidential Fellowship from the University of Manchester. Via our membership of the UK's HEC Materials Chemistry Consortium, which is funded by EPSRC (EP/L000202), this work used the ARCHER UK National Supercomputing Service (http://www.archer.ac.uk).

Supporting Information Available: Supercell convergence data, as well as further 
breakdown of the modal contributions to the lattice thermal conductivity.

\section{References}

(1) Mizushima, K.; Jones, P. C.; Wiseman, P. J.; Goodenough, J. B. $\operatorname{LixCoO}_{2}(0<\mathrm{x}<-1)$ : A New Cathode Material for Batteries of High Energy Density. Mater. Res. Bull. 1980, $15,783-789$.

(2) Gibbard, H. High Temp., High Pulse Power Lithium Batteries. J. Power Sources 1989, 26, 81-91.

(3) Plichta, E.; Slane, S.; Uchiyama, M.; Salomon, M.; Chua, D.; Ebner, W.; Lin, H. An Improved $\mathrm{Li} / \mathrm{Li}_{\mathrm{x}} \mathrm{CoO}_{2}$ Rechargeable Cell. J. Electrochem. Soc. 1989, 136, 1865-1869.

(4) Takahashi, Y.; Gotoh, Y.; Akimoto, J.; Mizuta, S.; Tokiwa, K.; Watanabe, T. Anisotropic Electrical Conductivity in $\mathrm{LiCoO}_{2}$ Single Crystal. J. Solid State Chem. $2002,164,1-4$.

(5) Islam, M. S.; Fisher, C. A. Lithium and Sodium Battery Cathode Materials: Computational Insights Into Voltage, Diffusion and Nanostructural Properties. Chem. Soc. Rev. 2014, 43, 185-204.

(6) Sun, H.; Zhao, K. Electronic Structure and Comparative Properties of $\mathrm{LiNi}_{x} \mathrm{Mn}_{y} \mathrm{Co}_{z} \mathrm{O}_{2}$ Cathode Materials. J. Phys. Chem. C 2017, 121, 6002-6010.

(7) Ohzuku, T.; Makimura, Y. Layered Lithium Insertion Material of $\operatorname{LiNi}_{1 / 2} \mathrm{Mn}_{1 / 2} \mathrm{O}_{2}$. Chem. Lett. 2001, 30, 744-745.

(8) Du, T.; Xu, B.; Wu, M.; Liu, G.; Ouyang, C. Insight Into the Vibrational and Thermodynamic Properties of Layered Lithium Transition-Metal Oxides $\mathrm{LiMO}_{2}(\mathrm{M}=\mathrm{Co}$, Ni, Mn): A First-Principles Study. J. Phys. Chem. C 2016, 120, 5876-5882. 
(9) Goodenough, J. B.; Kim, Y. Challenges for Rechargeable Li Batteries. Chem. Mater. 2009, 22, 587-603.

(10) Tarascon, J.-M.; Armand, M. Materials for Sustainable Energy; World Scientific, 2011.

(11) Bandhauer, T. M.; Garimella, S.; Fuller, T. F. A Critical Review of Thermal Issues in Lithium-Ion Batteries. J. Electrochem. Soc. 2011, 158, R1-R25.

(12) Urban, A.; Seo, D.-H.; Ceder, G. Computational Understanding of Li-Ion Batteries. npj Comput. Mater. 2016, 2, 16002.

(13) Qian, X.; Gu, X.; Dresselhaus, M. S.; Yang, R. Anisotropic Tuning of Graphite Thermal Conductivity by Lithium Intercalation. J. Phys. Chem. Lett. 2016, 7, 4744-4750.

(14) Wei, Z.; Yang, F.; Bi, K.; Yang, J.; Chen, Y. Tunable Anisotropic Thermal Conductivity and Elastic Properties in Intercalated Graphite via Lithium Ions. J. Phys. Chem. C 2018, 122, 1447-1455.

(15) He, J.; Zhang, L.; Liu, L. Thermal Transport in Monocrystalline and Polycrystalline Lithium Cobalt Oxide. Phys. Chem. Chem. Phys. 21, 12192-12200.

(16) Momma, K.; Izumi, F. VESTA 3 for Three-Dimensional Visualization of Crystal, Volumetric and Morphology Data. J. Appl. Crystallogr. 2011, 44, 1272-1276.

(17) Cramer, C. J.; Truhlar, D. G. Density Functional Theory for Transition Metals and Transition Met. Chem. Phys. Chem. Chem. Phys. 2009, 11, 10757-10816.

(18) Monserrat, B.; Park, J.-S.; Kim, S.; Walsh, A. Role of Electron-Phonon Coupling and Thermal Expansion on Band Gaps, Carrier Mobility, and Interfacial Offsets in Kesterite Thin-Film Solar Cells. Appl. Phys. Lett. 2018, 112, 193903.

(19) Inaba, M.; Iriyama, Y.; Ogumi, Z.; Todzuka, Y.; Tasaka, A. Raman Study of Layered Rock-Salt $\mathrm{LiCoO}_{2}$ and Its Electrochemical Lithium Deintercalation. J. Raman Spectrosc. 1997, 28, 613-617. 
(20) Rao, K.; Benqlilou-Moudden, H.; Desbat, B.; Vinatier, P.; Levasseur, A. Infrared Spectroscopic Study of $\mathrm{LiCoO}_{2}$ Thin Films. J. Solid State Chem. 2002, 165, 42-47.

(21) Togo, A.; Chaput, L.; Tanaka, I. Distributions of Phonon Lifetimes in Brillouin Zones. Phys. Rev. B 2015, 91, 094306.

(22) Takahata, K.; Terasaki, I. Thermal Conductivity of $A_{x} B \mathrm{O}_{2}$-Type Layered Oxides $\mathrm{Na}_{0.77} \mathrm{MnO}_{2}$ and $\mathrm{LiCoO}_{2}$. Jap. J. Appl. Phys. 2002, 41, 763.

(23) Broido, D. a.; Malorny, M.; Birner, G.; Mingo, N.; Stewart, D. Intrinsic Lattice Thermal Conductivity of Semiconductors From First Principles. Appl. Phys. Lett. 2007, 91, 231922 .

(24) Skelton, J. M.; Parker, S. C.; Togo, A.; Tanaka, I.; Walsh, A. Thermal Physics of the Lead Chalcogenides PbS, PbSe, and PbTe From First Principles. Phys. Rev. B 2014, $89,205203$.

(25) Whalley, L. D.; Skelton, J. M.; Frost, J. M.; Walsh, A. Phonon Anharmonicity, Lifetimes, and Thermal Transport in $\mathrm{CH}_{3} \mathrm{NH}_{3} \mathrm{PbI}_{3}$ From Many-Body Perturbation Theory. Phys. Rev. B 2016, 94, 220301.

(26) Yan, B.; Lim, C.; Yin, L.; Zhu, L. Simulation of Heat Generation in a Reconstructed $\mathrm{LiCoO}_{2}$ Cathode During Galvanostatic Discharge. Electro. Acta 2013, 100, 171-179.

(27) Feng, X.; Ouyang, M.; Liu, X.; Lu, L.; Xia, Y.; He, X. Thermal Runaway Mechanism of Lithium Ion Battery for Electric Vehicles: A Review. Energy Stor. Mater. 2018, 10, $246-267$.

(28) Assat, G.; Glazier, S. L.; Delacourt, C.; Tarascon, J.-M. Probing the Thermal Effects of Voltage Hysteresis in Anionic Redox-Based Lithium-Rich Cathodes Using Isothermal Calorimetry. Nature Energy 2019, 4, 647-656. 
(29) Hertz, J. T.; Huang, Q.; McQueen, T.; Klimczuk, T.; Bos, J.; Viciu, L.; Cava, R. J. Magnetism and Structure of $\mathrm{Li}_{\mathrm{x}} \mathrm{CoO}_{2}$ and Comparison to $\mathrm{Na}_{\mathrm{x}} \mathrm{CoO}_{2}$. Phys. Rev. B 2008, r7., 075119.

(30) Kresse, G.; Furthmller, J. Efficient Iterative Schemes for Ab Initio Total-Energy Calculations Using a Plane-Wave Basis Set. Phys. Rev. B 1996, 54, 11169-11186.

(31) Tkatchenko, A.; Scheffler, M. Accurate Molecular Van Der Waals Interactions From Ground-State Electron Density and Free-Atom Reference Data. Phys. Rev. Lett. 2009, 102, 073005.

(32) Bučko, T.; Lebègue, S.; Ángyán, J. G.; Hafner, J. Extending the Applicability of the Tkatchenko-Scheffler Dispersion Correction via Iterative Hirshfeld Partitioning. The J. Chem. Phys. 2014, 141, 034114.

(33) Seo, D.-H.; Urban, A.; Ceder, G.; Others, Calibrating Transition-Metal Energy Levels and Oxygen Bands in First-Principles Calculations: Accurate Prediction of Redox Potentials and Charge Transfer in Lithium Transition-Metal Oxides. Phys. Rev. B 2015, 92, 115118.

(34) Okumura, T.; Yamaguchi, Y.; Shikano, M.; Kobayashi, H. Correlation of Lithium Ion Distribution and X-Ray Absorption Near-Edge Structure in O3-And O2-Lithium Cobalt Oxides From First-Principle Calculation. J. Mater. Chem. 2012, 22, 1734017348.

(35) Paier, J.; Marsman, M.; Hummer, K.; Kresse, G.; Gerber, I. C.; Ángyán, J. G. Screened Hybrid Density Functionals Applied to Solids. The J. Chem. Phys. 2006, 124, 154709.

(36) Togo, A.; Tanaka, I. First Principles Phonon Calculations in Mater. Sci. Scripta Mater. 2015, 108, 1-5. 